\title{
High-Power Long-Pulse Second Harmonic Generation and Optical Damage With Free-Running Nd: YAG Laser
}

\author{
Sébastian Favre, Thomas C. Sidler, and René-Paul Salathé, Senior Member, IEEE
}

\begin{abstract}
Frequency doubling with a free-running long-pulse Nd:YAG laser and LBO or KTP nonlinear crystals yields conversion efficiency of up to $17.5 \%$ and $162 \mathrm{~W}$ peak power in the second harmonic. This efficiency is obtained for $\mathbf{a ~ T E M}_{00}$ beam with rectangular temporal pulse shape of 50 to $400 \mu \mathrm{s}$. To our knowledge, this is the highest second-harmonic generation (SHG) efficiency reported for the long-pulse free-running configuration. The efficiency is limited by optical damage with much lower threshold than in the Q-switch domain. The damage is preceded by a saturation effect of the SHG efficiency. Both wavelengths (fundamental and second-harmonic) are necessary for the creation of the catastrophic damage. We present first evidence for a mechanism that involves creation of transient absorption centers by the second-harmonic radiation due to multiphoton absorption. Absorption of the fundamental wave at these centers leads to local heating and ultimately catastrophic damage.
\end{abstract}

Index Terms-Free running, gray tracks, KTP, laser, optical damage, second-harmonic generation (SHG).

\section{INTRODUCTION}

$\mathbf{M}$ OST of extra-cavity second harmonic generation (SHG) with Nd:YAG lasers have been performed so far with Q-switch lasers (pulses of several nanoseconds) as this type of laser can easily deliver the peak powers necessary to achieve good conversion efficiencies. For this mode of operation, efficiencies of more than $65 \%$ [1] and average powers as high than $100 \mathrm{~W}$ [2] have been reported. Usually, for CW SHG, the nonlinear (NLO) crystal is placed intra-cavity [3].

However, typical industrial applications such as cutting and drilling are based on free-running Nd:YAG lasers with pulses of $10-1000-\mu$ s width, since the material ablation rate is much higher in this region. The higher absorption of most materials at the second-harmonic wavelength permits achievement of higher processing yields. Also, it opens up new possibilities for processing of some "difficult" materials like copper (with, e.g., high infrared (IR) reflection) [4].

But frequency doubling with long-pulse lasers is more difficult and less efficient and no results have been reported so far for free-running Nd:YAG lasers. With respect to a very recent work of Mu and Ding [19], we investigated SHG at significantly larger pulsewidths and higher beam powers. The main problem

Manuscript received April 18, 2002; revised October 25, 2002. This work was supported by a Swiss Government grant (Minast).

The authors are with the Institute of Imaging and Applied Optics, Swiss Federal Institute of Technologies (EPFL), CH-1015 Lausanne EPF, Switzerland.

Digital Object Identifier 10.1109/JQE.2003.810764

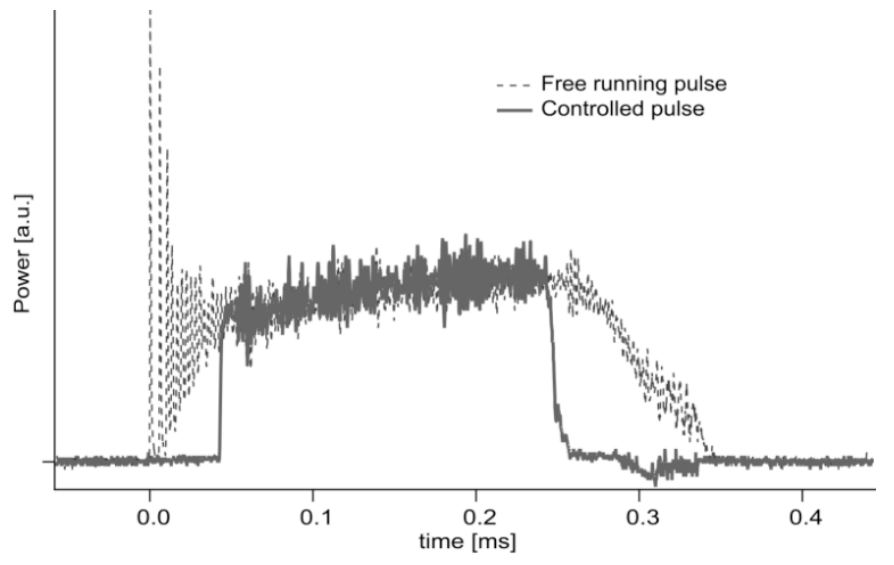

Fig. 1. Controlled pulse shape with optical shutter.

for an efficient nonlinear frequency conversion is the generation of a powerful beam at the fundamental wavelength (1064 $\mathrm{nm}$ ), characterized by high brightness and low divergence. Furthermore, an extra-cavity SHG crystal configuration has to be chosen, to avoid high thermal load and related problems of beam degradation [5].

In this mode, the appearance of optical damage in the nonlinear crystals limits SHG efficiency. The damage mechanism is clearly material dependent, but fluence, energy, and pulsewidth are other important factors. To date, only short pulse (Q-switched) or continuous-wave (CW) damage thresholds have been reported in the literature [1]. Several mechanisms have been proposed to explain damage creation [6]-[12]. Self-focusing, breakdown, or other high-intensity processes can be excluded from the experimental conditions in our work based on much lower intensities. Creation of color centers or "gray tracks" have been reported for CW [6] and Q-switch lasers [7] and it has been demonstrated that second-harmonic radiation alone is responsible for the formation of such defects [8]. Appearance and disappearance of such absorption centers can show different dynamic behaviors, and the temporary character and the nature of the centers are still under investigation [9]-[12].

In this paper, we report and analyze new results on long-pulse SHG with KTP (potassium titanyl phosphate $\left[\mathrm{KTiOPO}_{4}\right.$ )] and LBO (lithium triborate $\left.\left(\mathrm{LiB}_{3} \mathrm{O}_{5}\right)\right]$ nonlinear crystals and determine the catastrophic optical damage threshold for different pulsewidths. The long-pulse damage threshold measured in the frame of this work is up to 50 times lower than the value observed for Q-switch lasers. Creation of temporary color centers 


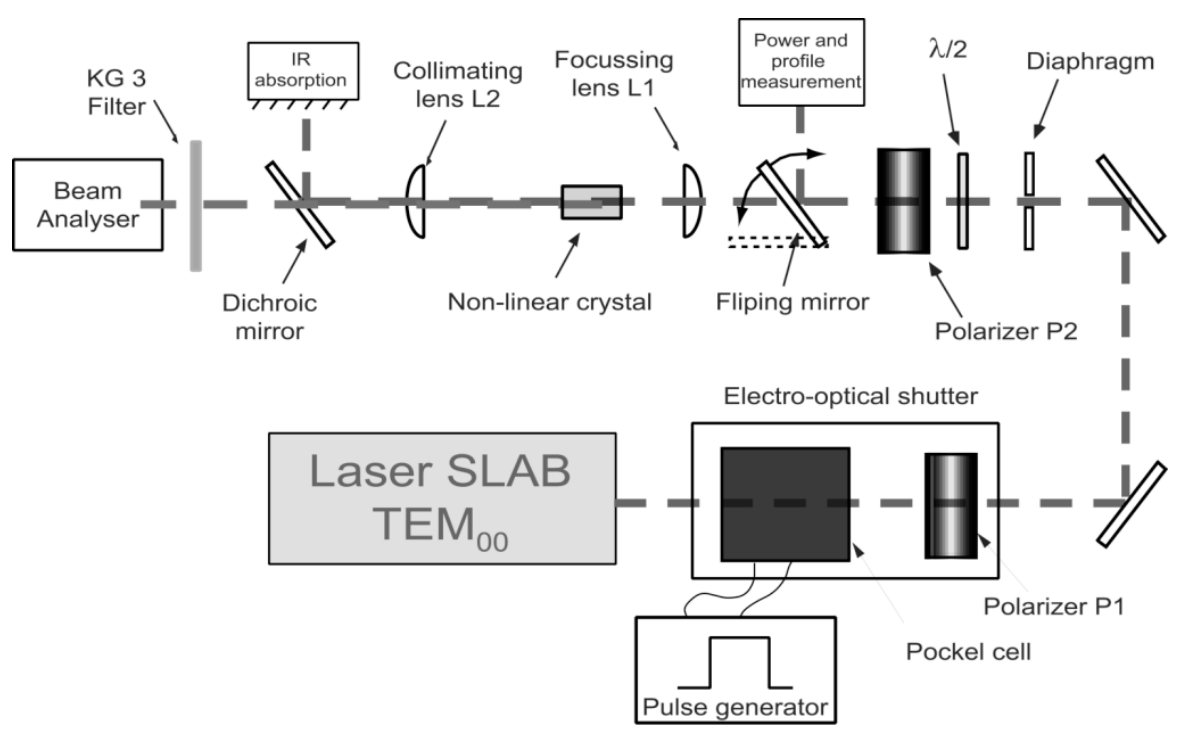

Fig. 2. Experimental setup. The orientation of the polarizer P2 and the half-wave plate permit fine adjustment of the laser power incident on the nonlinear crystals.

by the second-harmonic radiation is identified as the most probable mechanism at the starting point of the degradation. Then we show that absorption of the fundamental wave at the centers is the most likely process leading to local heating, and finally, catastrophic damage.

\section{SetUP AND DESIGN}

To provide the high-brightness laser beam, we use a zigzag slab (parallelepiped medium geometry) Nd:YAG crystal, pumped on two sides by $\mathrm{Kr}$ flash lamps [18]. This geometry and the Brewster-angle cut crystal are ideally suited to obtain a linearly polarized nearly $\mathrm{TEM}_{00}$-mode laser beam (polarization ratio $>500: 1)$ necessary for the nonlinear process, without the problems of polarization losses of typical rod lasers. Another advantage of zigzag propagation in the medium is minimization of the thermal lens effects [13]. Selection of the fundamental mode is done with an intra-cavity diaphragm. To increase overlap between the laser mode and the pumped crystal volume, a triple passage in a " $z$ " configuration was implemented. Furthermore, the adjunction of a specifically calculated intra-cavity cylindrical telescope results in an elliptical beam in the laser medium which leads to higher interaction volume and which gives a circular beam on the flat output mirror rendering the laser output free of astigmatism [14]. This laser produces a quasi-CW 1064-nm fundamental mode beam of $0.1-5 \mathrm{~ms}$ pulsewidth with a peak power of $1-2 \mathrm{~kW}$ and a $\mathrm{TEM}_{00}$ beam quality. A more detailed description of the system has been presented elsewhere [18].

The spatial beam profile of $200-\mu$ s $300-\mathrm{mJ}$ pulses was analyzed at a repetition rate of $10 \mathrm{~Hz}$ and revealed $M^{2}<1.7$. As the laser was working in a fundamental mode, a well-developed damped spiking with eight to ten times higher peak power than the average pulse power was observed at the leading edge of the pulse (see Fig. 1). Since such high-power spikes could result in crystal damage, an extra-cavity electrooptical shutter consisting of a Pockels cell followed by a polarizer was inserted. In this way, the generation of purely rectangular pulses with minimal losses and a rise time of $1 \mu \mathrm{s}$, with controllable width from 10 to

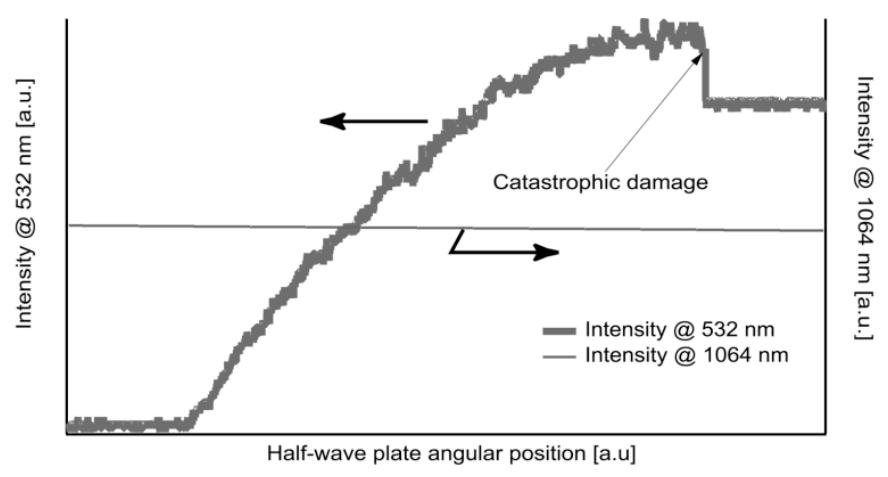

Fig. 3. SHG and damage creation through adjustment of incident polarization angle.

$1000 \mu$ s was obtained. Fig. 1 shows the result of this procedure. The original pulse with its spiking and the resultant rectangular shaped pulse are compared. In the shutter off-state, the residual transmission is less than $0.08 \%$.

The experimental setup used for SHG determination and analysis is presented in Fig. 2. The 1064-nm laser beam, after clipping of the leading edge spikes, is directed via two mirrors, a diaphragm, a half-wave plate, and a dielectric polarizer onto lens $L 1$. The half-wave plate and the polarizer $\mathrm{P} 2$ allow precise control of the pulse power without changing either the pump energy or the beam shape. The light is then focused onto the crystal by lens $L 1$, recollimated by lens $L 2$ and directed onto a beam analyzer. A dichroic mirror is used to direct the remaining IR beam light onto an absorber.

The reproducible temporal and spatial beam quality permits precision control of the intensity and divergence at the focal point of $L 1$ to be optimally adapted to the properties (e.g., acceptance angles) of the different nonlinear crystals.

The laser was then operated at a low pulse-repetition rate (i.e., low mean power) in order to prevent excessive thermal loading of the nonlinear crystals, leading to degradation in beam quality. In addition, all experiments were performed under temperature-controlled conditions with the nonlinear crystals mounted on a Peltier element or on a precision micro-heater. 


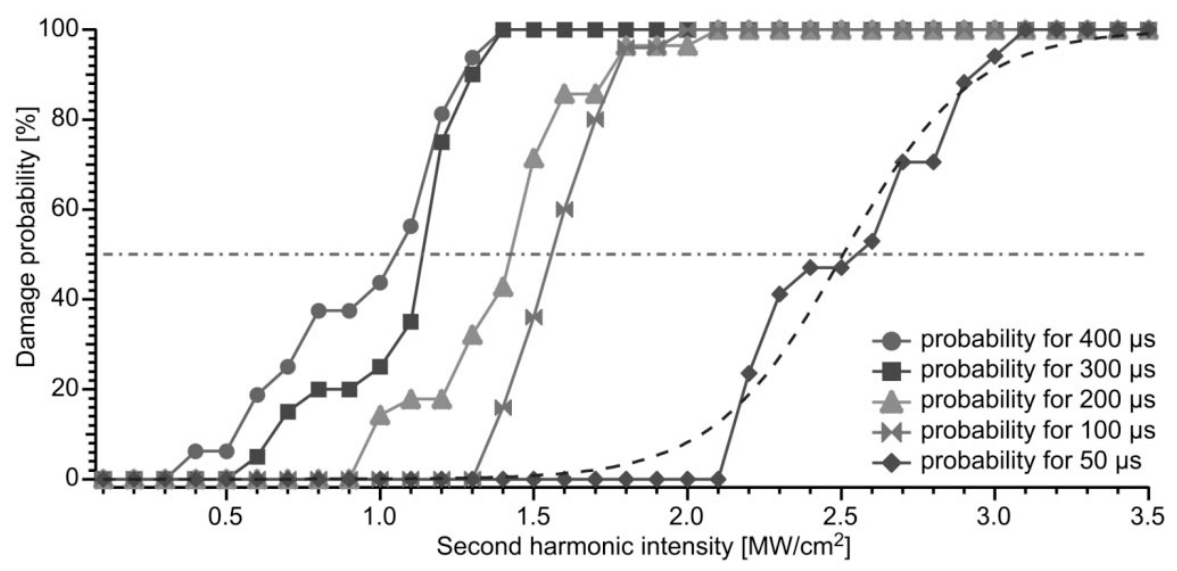

Fig. 4. Probability of optical damage versus second-harmonic intensity for different pulsewidths.

These holders were placed on a four-axis stage (two rotational and two translation), which permit fine adjustment of the crystal position and control of the exact interaction location. To satisfy the plane-wave approximation, divergence of the beam must be negligible inside the crystal, i.e., the Rayleigh range must be large compared to the crystal length. For the crystal lengths of $1-15 \mathrm{~mm}$ used in our experiments, the laser was focused to a spot diameter of $274 \mu \mathrm{m}$ (focusing lens $L 1=300 \mathrm{~mm}$ in Fig. 2), yielding a Rayleigh length $z_{R}$ of $33 \mathrm{~mm}$.

\section{Catastrophic Damage}

SHG conversion efficiency is generally limited by catastrophic optical damage occurring at higher intensities close to the exit facet of the nonlinear crystal. To investigate this problem, the following procedure was applied. The intensity (respectively power) of the fundamental wave was kept constant at typically $1.7 \mathrm{MW} / \mathrm{cm}^{2}$ and the generated green intensity was varied by adjusting the input polarization through rotation of the half wave plate. For these experiments, the polarizer has been placed in front of the half-wave plate (cf. Fig. 2). The green intensity was increased slowly (to ensure a thermal equilibrium within the nonlinear crystal). Fig. 3 shows the measured intensities for the fundamental and second-harmonic wave as a function of the angular position of the half-wave plate. First, the second-harmonic power increases with increasing angle, then it saturates. A distinct decrease in second-harmonic power indicates occurrence of catastrophic damage at intensity levels of typically $1-2 \mathrm{MW} / \mathrm{cm}^{2}$ of green light. Optical inspection of the crystals after the experiments has shown that the damaged zone was always located close to the exit facet of the nonlinear crystal.

Basically, the damage could be produced by the fundamental or second-harmonic waves alone or by the presence of both wavelengths. Experiments at the fundamental wavelength alone with $200-\mu$ s pulses showed damage threshold above 40 $\mathrm{MW} / \mathrm{cm}^{2}$, corresponding to intensities more than ten times higher than the threshold measured in the presence of both wavelengths. Similarly, for second-harmonic radiation alone, the damage threshold is at least an order of magnitude higher. This value has been measured with the same pulse parameters (pulsewidth, power, etc ...), with a green beam issued from

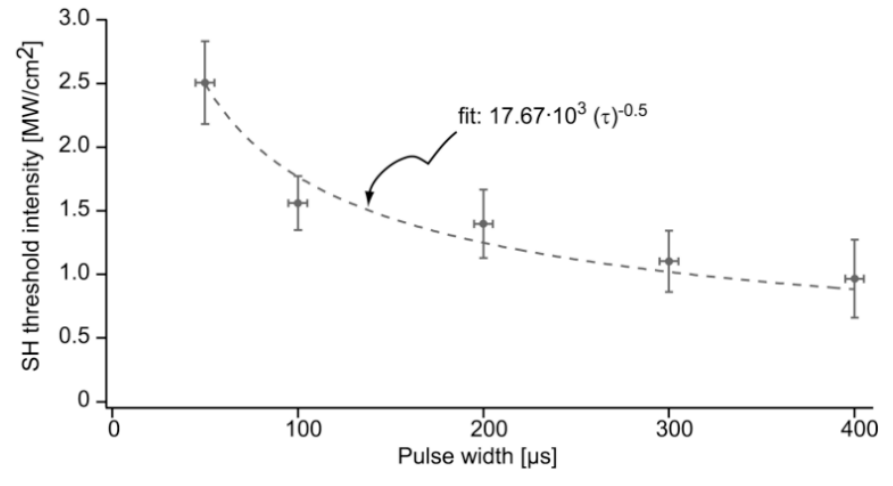

Fig. 5. Damage threshold for green light versus pulsewidth.

the same system, focused onto another crystal. A threshold of more than $40 \mathrm{MW} / \mathrm{cm}^{2}$ has been measured. Therefore, we conclude that the presence of both wavelengths leads to a much lower damage threshold than for each wavelength alone. Moreover, the second-harmonic intensity is the key parameter for its creation.

The same kind of experiment has also been performed with temperature controlled phase mismatch, with identical results. All the experiments for the determination of the optical damage threshold versus green intensity have been repeated some 20 times and the statistical mean values are reported. No dependence has been observed for different crystal lengths.

Fig. 4 shows the damage probability versus second-harmonic intensity for different pulsewidths. For each pulsewidth, the intensity range for a damage to occur is limited to $\pm 0.5 \mathrm{MW} / \mathrm{cm}^{2}$. Since avalanche ionization processes usually show a much larger spread of the destruction threshold [16], we suppose rather a multiphotonic ionization at the origin of the degradation in our case.

Moreover, the result presented in Fig. 3 suggests that the process is started by the second-harmonic light through multiphotonic ionization resulting in a seed. Localized avalanche ionization due to the fundamental wave can then take place in such affected zone.

The damage intensity threshold, defined by the $50 \%$ failure rate, versus pulsewidth is presented in Fig. 5. This threshold decreases with pulsewidths according to a $\tau^{-1 / 2}$ law. Such 


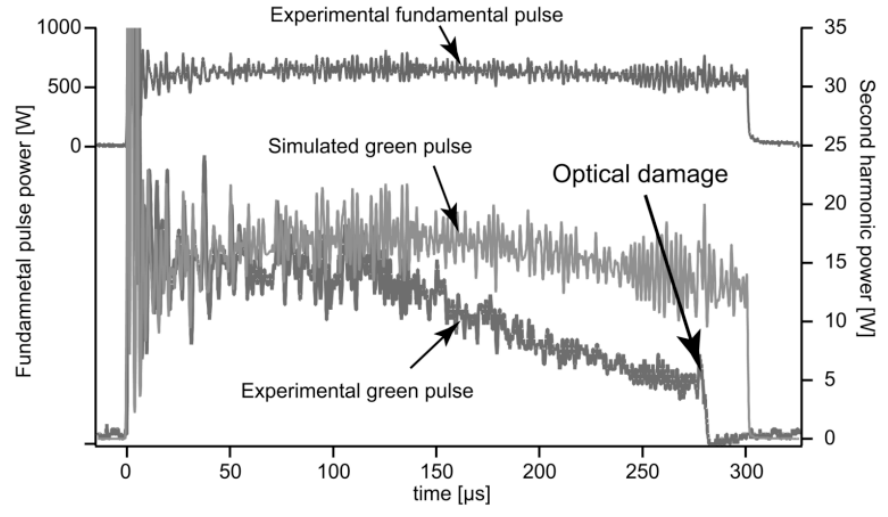

Fig. 6. Fundamental and green pulse shapes, at power levels resulting in a damage.

behavior is typically observed for two-photon absorption processes [17].

Furthermore, an extrapolation of this fit over several orders of magnitude results in good agreement with the threshold values reported for nanosecond (Q-switch) radiations [1] and picoseconds radiations [17].

\section{GRAY TRACK}

Investigation of the temporal shape for the infrared and green laser pulses, resulting in optical damage, is seen in Fig. 6. The comparison of the experimentally measured green pulse power with the calculated second-harmonic pulse power based on the plane-wave approximation reveals that the generated secondharmonic intensity significantly decreases in the second half of the pulse preceding optical damage.

This effect is not present for parameters far from damage threshold. This loss in second-harmonic power may be related to a local thermal load in the crystal, bringing it out of its critical temperature phase match, or simply to an absorption of the fundamental and/or second-harmonic beam. These phenomena are strongly related and difficult to separate, as any absorption will inevitably result in an increased thermal load. As the green light intensity is the highest on the output facet of the crystal, it is quite common that damage occurs preferentially in this area.

With the optical setup shown in Fig. 7, the SHG effective area is observed with an off-axis probe laser beam (at $670 \mathrm{~nm}$ ) focused on the output facet of the crystal. To ensure the highest sensitivity, the spot diameter of the probe laser has been chosen close to the diameter of the generated green beam.

Fig. 8 shows the transmission of the $670-\mathrm{nm}$ probe beam and the infrared power as a function of time for different secondharmonic beam energies at intensities below the catastrophic damage threshold. The transmission decreases during the laser pulse of $200 \mu$ s durations. This decrease is more pronounced and becomes nonlinear at higher second-harmonic energies. Note that normally, gray-track spectra show absorptions over a wide wavelength range, e.g., as observed in gray-track generated by external electric-field application [10]. We, therefore, suspect that the decreased $670-\mathrm{nm}$ light transmission corresponds to an increased absorption at the second-harmonic wavelength (as also suggested in Fig. 6) and at the fundamental laser wavelength. At the end of the pulse, the transmission of the probe

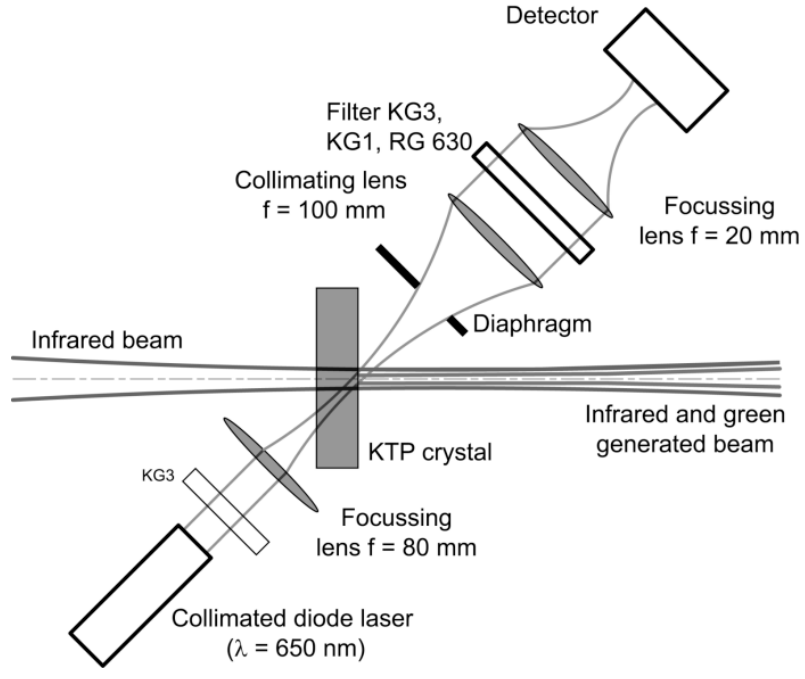

Fig. 7. Probe-beam experiment setup.

beam increases at a different time constant and returns to the original value before the next pulse is triggered. This behavior clearly indicates the transient nature of the induced absorption centers. The dynamics of this color center creation and relaxation need further investigations, as both seem to be nonlinear.

Considering catastrophic damage, it is evident that absorption of the green light alone would not be able to generate the thermal damage as its power is "low" (10\%-20\% of the infrared, a maximum of $160 \mathrm{~W}$ ) and it therefore seems reasonable that the damage is rather generated through absorption of the 1064-nm wavelength, rather than absorption of the 532-nm beam.

\section{SHG EFFICIENCY}

In the plane-wave approximation, the second-harmonic conversion efficiency is given by [1]

$$
\eta=\frac{P_{2 \omega}}{P_{\omega}}=\tanh ^{2}\left\{\sqrt{l^{2} K I \frac{\sin ^{2}\left(\frac{\Delta k l}{2}\right)}{\left(\frac{\Delta k l}{2}\right)^{2}}}\right\}
$$

with

$$
K=2\left(\frac{\mu_{0}}{\varepsilon \varepsilon_{0}}\right)^{3 / 2}\left(\frac{2 \pi c}{\lambda_{\omega}}\right)^{2} d_{\mathrm{eff}}^{2}
$$

where $P_{i \omega}$ represents, respectively, the power of the fundamental $(i=1)$ and SHG beams $(i=2), I$ the infrared incident intensity, $l$ the length of the NLO crystal, $\Delta k$ a phase-mismatch parameter, and $K$ a constant proportional to $d_{\text {eff }}$, the nonlinear coefficient of the crystal (2). For weak conversion efficiency, the pump depletion can be neglected and the behavior is linear, given by the approximation $\tanh (x) \approx x[15]$.

As an example, Fig. 9 shows the second-harmonic efficiency as a function of fundamental incident intensity for a 10 -mm-long KTP crystal and 200- $\mu$ s laser pulses. The efficiency increases linearly with intensity up to $\approx 0.5 \mathrm{MW} / \mathrm{cm}^{2}$, to around, i.e., $6 \%$. The slope of the curve at low intensity has been used to determine the nonlinear coefficient of the investigated material. For higher incident intensities, the experimental 


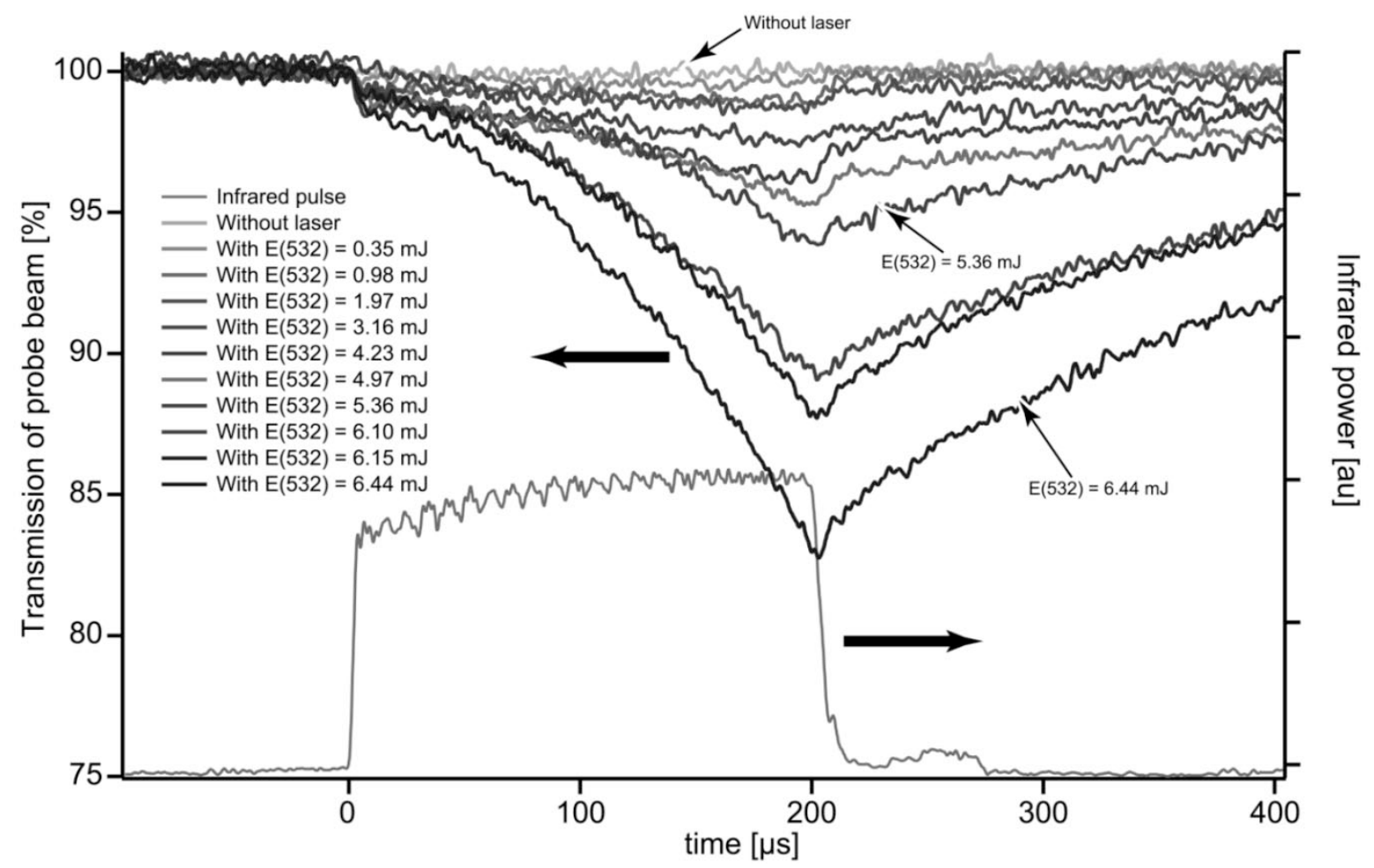

Fig. 8. Transmission of the probe beam for 200- $\mu$ s pulses with different SHG efficiencies. Curves from top to down correspond to an increase of second-harmonic energy as given in the insert.

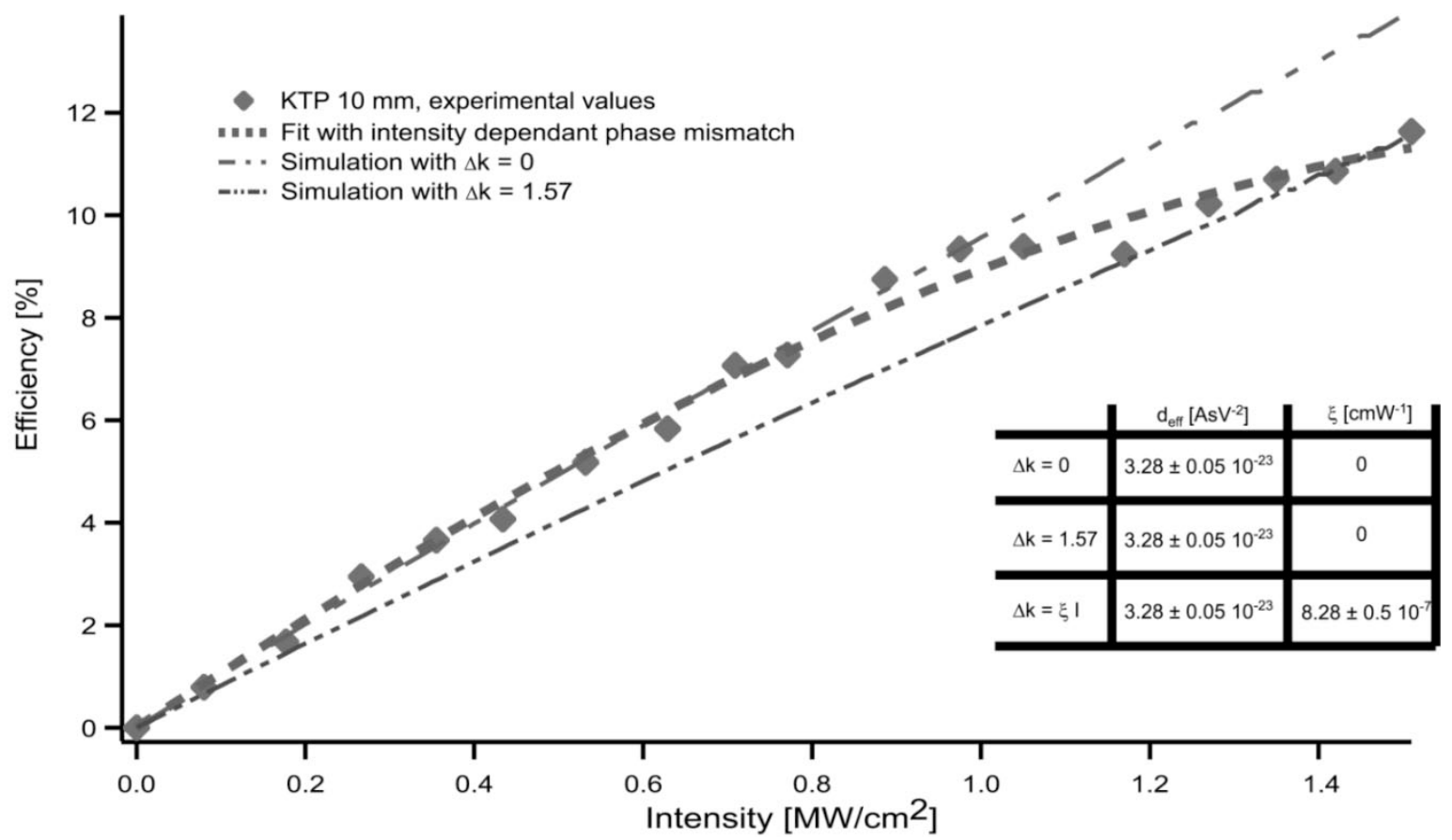

Fig. 9. Theoretical and measured SH-efficiencies for a 10-mm-long KTP crystal as a function of incident laser intensity. Intensity dependant phase mismatch parameters are indicated in the insert.

curve diverges from the small signal linear behavior and tends to saturate at intensities above $1 \mathrm{MW} / \mathrm{cm}^{2}$.

The interpretation of the nonlinear behavior at high intensities shown in the Fig. 9 can be explained by the directly observed transitory absorption affecting the SHG efficiency above $0.5 \mathrm{MW} / \mathrm{cm}^{2}$. The determination of the nonlinear coefficient $d_{\text {eff }}$ must be made by an analysis of the behavior at low intensi- ties, a linear fit of the complete experimental results could lead to a misleading determination of the nonlinear coefficient $d_{\mathrm{eff}}$.

This deviation from the ideal linear behavior has been observed to a different degree with all the different NLO crystals under investigation. As the laser parameters such as beam quality and temporal profile were strictly controlled, beam degradation could not be involved. Thus, this deviation is 


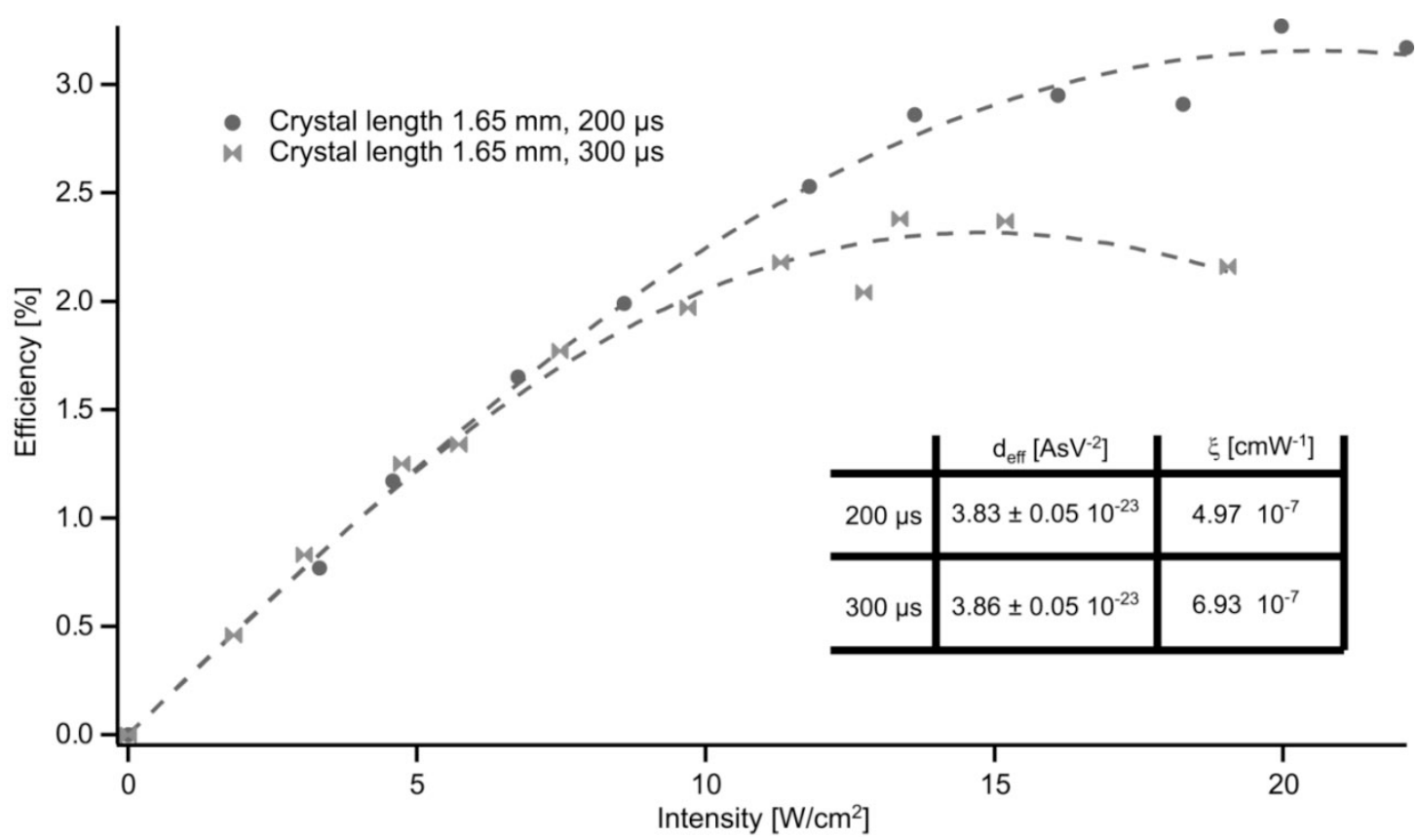

Fig. 10. Second-harmonic -efficiency versus 1064-nm intensity for a 1.65-mm-long KTP crystal and two different pulsewidths. Modeling results based on (3) with an intensity-dependent phase mismatch $\xi$ parameter are compared to experimental measurements.

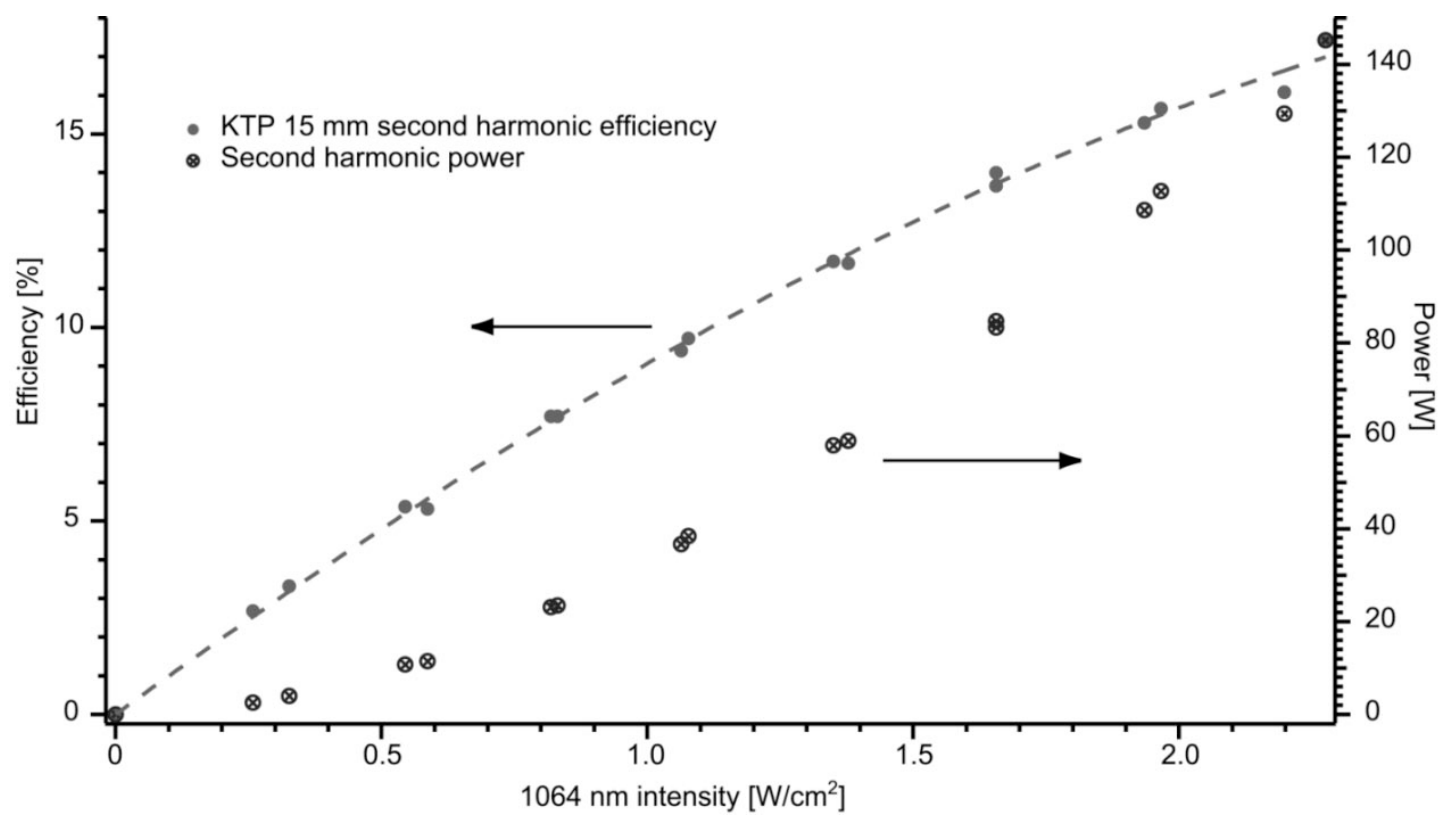

Fig. 11. Conversion efficiency and second-harmonic power versus intensity for a 15-mm-long KTP crystal and 200- $\mu$ s pulses.

clearly related to the interaction of the laser and the nonlinear medium and is the result of a power loss due to permanent or transitory absorption and/or a thermal phase-mismatch in the crystal (resulting from heat deposition).

The experimental behavior at higher intensities can no longer be described by the linear low conversion efficiency approximation. As angles and temperature of the crystal are continuously adjusted during all measurements, a mean phase mismatch can be excluded. Moreover, as shown in Fig. 9, a constant phase mismatch cannot fit the experimental behavior. To correctly take into account this intensity dependent effect, a heuristic intensity-dependent phase-mismatch $\Delta k=\xi I$ has been introduced, originating from the above explained (IR or SHG beam) intensity-dependent absorption. According to (1), the modified efficiency $\eta^{\prime}$ is then given by

$$
\eta^{\prime}(I)=\frac{P_{2 \omega}}{P_{\omega}}=\tanh ^{2}\left\{\sqrt{l^{2} K I \frac{\sin ^{2}\left(\frac{\xi I l}{2}\right)}{\left(\frac{\xi I l}{2}\right)^{2}}}\right\}
$$

The phenomenological phase-mismatch parameter $\xi$ permits a correct fitting of the results for all different lengths and types of crystals but also different pulsewidths for a given material, as is shown in Fig. 10. 


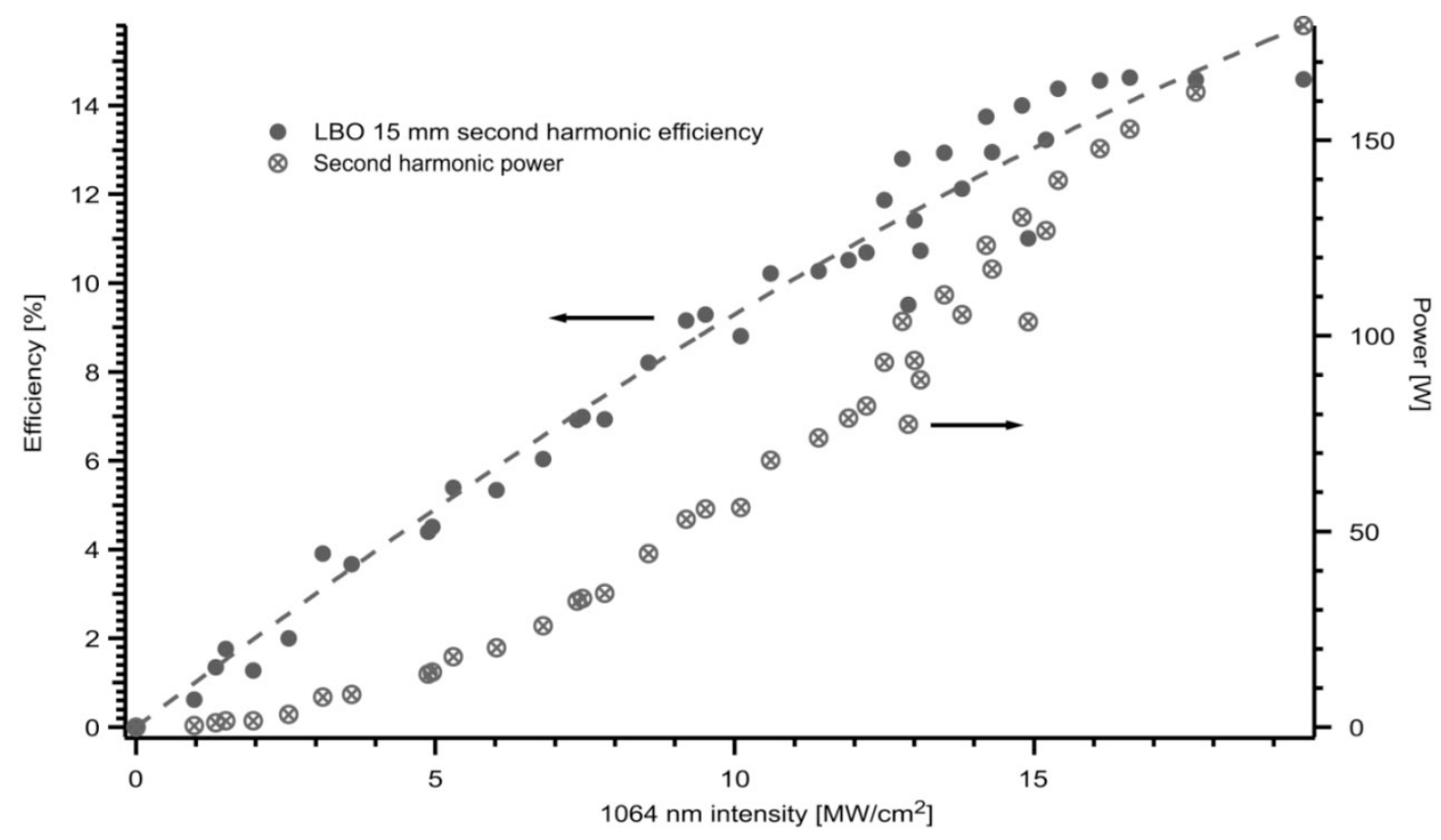

Fig. 12. Conversion efficiency and second-harmonic power versus intensity for a $15-\mathrm{mm}$-long LBO crystal and $200 \mu$ s pulses.

\begin{tabular}{|c|c|c|c|c|c|c|}
\hline $\begin{array}{l}\text { Infrared } \\
\text { radiation }\end{array}$ & $\stackrel{\text { SHG }}{(1064 \mathrm{~nm})}$ & $\begin{array}{c}\text { Green } \\
\text { radiation }\end{array}$ & (1532 nm) & $\begin{array}{l}\text { Color center } \\
\text { (2 green photon } \\
\text { absorption) }\end{array}$ & $\begin{array}{c}\text { Absorption of } \\
\text { the IR radiation }\end{array}$ & $\begin{array}{l}\text { Thermal } \\
\text { damage }\end{array}$ \\
\hline
\end{tabular}

Fig. 13. Damage mechanism in the long-pulse domain.

The parameter $\xi$ depends on the individual crystals and its introduction is very helpful for explaining the results and extraction of the corrected nonlinear coefficient $d_{\text {eff }}$, independently of the pulsewidth and the individual crystals.

Figs. 11 and 12 illustrate the best conversion efficiencies and resulting power at the second-harmonic wavelength versus incident infrared intensity for 15-mm-long KTP and LBO crystals.

The maximum SHG efficiency obtained without optical damage with a 15-mm-long crystal was $14.6 \%$ with LBO and $17.4 \%$ with KTP. Under these conditions we obtained for pulses of $200-\mu$ s duration up to 145 and $162 \mathrm{~W}$ of peak power and pulses energies of 32.4 and $29.0 \mathrm{~mJ}$, respectively. Note that different optimal input conditions are necessary, as the nonlinear coefficients of these two crystals are different.

Note that the intensity-dependent phase-mismatch parameter is several orders of magnitude $\left(10^{3}\right)$ lower for the LBO crystals than for the KTP crystals.

The results for the nonlinear coefficient $d_{\text {eff }}$ we obtained, with their relative range of error, are in good agreement with manufacturers data and the values reported in the literature (e.g., [1]).

\section{CONCLUSIONS}

First results and analysis of SHG with a free-running Nd:YAG slab laser and LBO and KTP crystals are presented. The SHG efficiency is influenced by the pulsewidth in this regime; a sublinear increase with laser intensity is observed at higher intensities with respect to the ideal phase-matched case and the total power conversion is ultimately limited by catastrophic damage at the output facet.

Time- and space-resolved transmission measurements reveal a transient absorption close to the exit facet of KTP nonlinear crystals under typical SHG conditions. We suspect that this absorption is induced by color centers generated by the second-harmonic radiation via a multiphoton process. At sufficiently high second-harmonic intensity and color center density, absorption of the unconverted infrared laser radiation leads to thermal catastrophic damage. The scheme shown in Fig. 13 summarizes the damage generation mechanism in the long-pulse domain. This scheme could also match with the Q-switch (nanoseconds) laser damage threshold. However, in this case, the intensity of the generated second-harmonic light is at a much higher level. Absorption of the second-harmonic radiation alone may then be able to induce directly the thermal damage itself.

The SHG efficiency loss observed toward the end of the laser pulses and the sublinear behavior of the generated second-harmonic intensity as a function of incident laser power can be related directly to the appearance of these absorption or color centers. The introduction of an intensity dependent phase-mismatch parameter allows to model the evolution of SHG efficiency as a function of incident laser power and to predict the saturation effects occurring at higher intensities in a quantitative way. Second-harmonic peak powers of 146 and $162 \mathrm{~W}$ at conversion efficiencies of $14.6 \%$ and $17.4 \%$, respectively, have been achieved for 15-mm-long KTP and LBO crystals. 


\section{ACKNOWLEDGMENT}

The authors gratefully acknowledge "Cristal Laser SA" (France) for providing a sufficient number of crystals necessary for these experiments.

\section{REFERENCES}

[1] W. Koechner, Solid-State Laser Engineering, 5th ed. Berlin, Germany: Springer-Verlag, 1996, ch. 10, pp. 582-620.

[2] S. P. Velsko et al., " $100 \mathrm{~W}$ average power at $0.53 \mu \mathrm{m}$ by external frequency conversion of an electro-optically $Q$-switched diode-pumped power oscillator," Appl. Phys. Lett., vol. 64, no. 23, pp. 3086-3088, June 1994.

[3] K. Koch and G. T. Moore, "Singly resonant cavity-enhanced frequency tripling," J. Opt. Soc. Amer. B, vol. 16, no. 3, pp. 448-459, Mar. 1999.

[4] S. Favre, T. Sidler, and R. P. Salathé, "High power second harmonic generation with free running ND.YAG slab laser for micro-machining applications," in Proc. SPIE, vol. 4088, Laser Precision Microfabrication, 2000, pp. 195-198.

[5] V. Pasiskevicius et al., "Efficient Nd: YAG laser frequency doubling with periodically poled KTP," Appl. Opt., vol. 37, no. 20, pp. 7116-7119, Oct. 1998.

[6] J. K. Tyminski, "Photorefractive damage in KTP used as second-harmonic generator," J. Appl. Phys., vol. 70, no. 10, pp. 5570-5576, Nov. 1991.

[7] N. Kuzuu et al., "Laser-induced bulk damage in various types of vitreous silica at 1064, 532, 355 and $266 \mathrm{~nm}$ : Evidence of different damage mechanisms between 266-nm and longer wavelengths," Appl. Opt., vol. 38, no. 12, pp. 2510-2515, Apr. 1999.

[8] G. M. Loiacono et al., "Laser damage formation in $\mathrm{KTiOPO}_{4}$ and $\mathrm{KTiOPO}_{4}$ crystals: Grey tracks," J. Appl. Phys., vol. 72, no. 7, pp. 2705-2712, Oct. 1991

[9] B. Boulanger et al., "Optical studies of laser-induced gray-tracking in KTP," IEEE J. Quantum Electon., vol. 35, pp. 281-286, Mar. 1999.

[10] M. N. Satiyanarayan et al., "Evidence for the presence of remnant strain in grey-tracked $\mathrm{KTiOPO}_{4}$," Appl. Phys. Lett., vol. 67, no. 19, pp. 2810-2812, Nov. 1995.

[11] J. P. Fève et al., "Repetition rate dependence of gray-tracking in $\mathrm{KTiOPO}_{4}$ during second-harmonic generation at $532 \mathrm{~nm}$," Appl. Phys. Lett., vol. 70, no. 3, pp. 277-279, Jan. 1997.

[12] B. Boulanger et al., "Study of $\mathrm{KTiOPO}_{4}$ gray-tracking at 1064, 532 and 355 nm," Appl. Phys. Lett., vol. 65, no. 19, pp. 2401-2403, Nov. 1994.

[13] T. Sidler and P. Verboven, "Optimal design of a flashlamp pumped free running Nd: YAG slab laser for harmonic frequency generation," in Proc. 11th Int. Congress Lasers, LASER 93, Lasers in Engineering, W. Waidelich, Ed. Berlin, Germany: Springer-Verlag, 1993, pp. 50-58.

[14] T. Sidler, private communication, 2002.

[15] T. A. Driscoll et al., "Efficient 2nd-harmonic generation in KTP crystals," J. Opt. Soc. Amer. B, vol. 3, no. 5, pp. 683-686, May 1986.

[16] X. Liu et al., "Laser ablation and micromachining with ultrashort laser pulses,” IEEE J. Quantum Electron., vol. 33, pp. 1706-1716, Oct. 1997.

[17] L. Carrion et al., "Gray-track damage in potassium titanyl phosphate under a picosecond regime at $532 \mathrm{~nm}$," Appl. Phys. Lett., vol. 77, no. 8, pp. 1074-1076, Aug. 2000.

[18] T. C. Sidler, "High efficiency laser resonators for high brightness beam generation, with application to micro-material processing and nonlinear process," in Proc. SPIE, vol. 4629, 2002, pp. 49-57.

[19] X. Mu and Y. J. Ding, "Third- and Second-harmonic generation from $1.319-\mu \mathrm{m} \mathrm{Nd}$ : YAG laser in one $\mathrm{KTiOPO}_{4}$ crystal," in Proc. CLEO 2002, 2002.

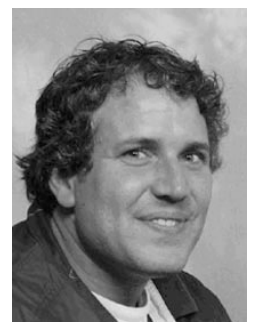

Sébastian Favre was born in La Chaux-de-Fonds, Switzerland, in 1968. He received the diploma in electronic physics from the University of Neuchâtel, Neuchâtel, Switzerland, in 1995, where he worked on holography and electronics sensors, and the Ph.D. degree from the Institute of Imaging and Applied Optics, Swiss Federal Institute of Technology (EPFL), Lausanne, Switzerland, in 2001.

As the Chair of Applied Optics, University of Neuchâtel, his work consisted of the application of liquid-crystal displays as phase modulators. Later, at EPFL, he worked in collaboration with industrial partners in the field of laser microprocessing. He also developed a high-power long-pulse ultraviolet laser source, based on the third-harmonic generation of a Nd:YAG slab laser through appropriate nonlinear appropriates mediums. In 2001, he was in the Laser Microprocessing Track, Data Storage Institute, at the National University of Singapore, for one year, where he worked on laser-matter interactions as visiting scientist. Since March 2002, he has been with the Biomedical Photonics Group at EPFL, working in the field of microscopy and optical low-coherence tomography.

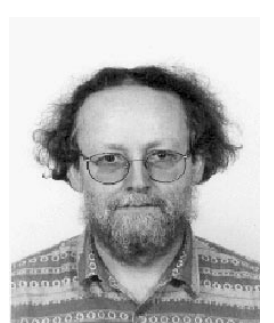

Thomas C. Sidler was born in Bienne, Switzerland. He received the diploma in physics and the Ph.D. degree for work in the area of Vk-centers in cesium iodide, both from the University of Neuchâtel, Neuchâtel, Switzerland, in 1971 and 1976, respectively.

From 1971 to 1976, he was an Assistant at the Physics Institute, University of Neuchâtel, working in solid-state physics. From 1976 to 1982, he conducted research in the areas of fiber optics, holographic interferometry, optical pattern recognition, and signal processing at the Microtechnics Institute, University of Neuchâtel. From 1982 to 1986, he was with research group management at Asulab SA, Neuchâtel, Switzerland, working on high-stability $\mathrm{CO}_{2}$ lasers for metrology and Nd-YAG high-power, high-beam-quality lasers for precision material processing. He joined the Institute of Applied Optics, Swiss Federal Institute of Technology (EPFL), Lausanne, Switzerland, in 1992, where his research focused on laser source development (diode-pumped solid-state lasers, flash-lamp pumped frequency-doubled Nd-YAG slab lasers), laser applications in material processing (high-precision laser cutting, high-reliability industrial welding, etc.), and other research activities based on lasers and microtechnics. During 1996-1997, his attention focused on the design, development, and realization of a 355-nm frequency-tripled Nd-YAG laser source, in the frame of an Incoherent Doppler Wind Lidar project.

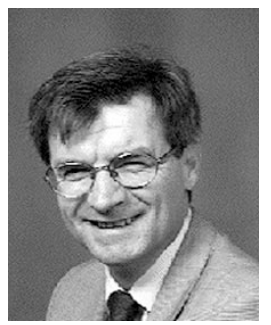

René-Paul Salathé (SM'88) received the M.S., Ph.D., and Habilitation (Privatdozent) degrees in 1970, 1974, and 1979, respectively, from the University of Bern, Bern, Switzerland, where he was actively engaged in research on semiconductor lasers.

He is a Professor of Applied Optics at the Swiss Federal Institute of Technology (EPFL), Lausanne, Switzerland. During 1977-1978, he was with Bell Telephone Laboratories, Murray Hill, NJ, working in the field of integrated optics. From 1978 to 1983, he directed a research group engaged on laser processing at the University of Bern. In 1984, he became Head of the "Technology" section, and in 1985, Head of the division "Material Testing and Technology," both at the research and development center of Swiss Telecom (PTT). During 1989-2001, he was Director of the Institute of Applied Optics (Institut d'Optique Appliquée), Micro-engineering Department, EPFL, and during 1995-1998, was Head of the Microengineering Department. During the winter semester 1998/1999, he was on a sabbatical leave at the Center for Biological and Medical Engineering, University of Texas, Austin. Since 2002, he has been heading the Institute "Imagerie et Optique Appliquée" (IOA), Faculty of Engineering Sciences and Techniques, EPFL.

Dr. Salathe is is a member of the Swiss Physical Society, the Swiss Society for Optics and Electron Microscopy, the European Optical Society, the Optical Society of America, and a member of the Board of the Swiss Electrotechnical Association. 